\title{
Können Physiotherapeuten klinisch relevante Veränderungen des Straight-Leg-Raise-Tests bei Probanden mit subakuten/akuten Beschwerden feststellen? \\ Pilotstudie
}

\author{
Are Physiotherapists Able to Determine Clinicially Important Changes of \\ the Straight Leg Raise Test in Subjects with Subacute/Acute Complaints? \\ Pilot Study
}

Autoren

Institute
M. Bräuer ${ }^{1}$, T. Schöttker-Königer ${ }^{1}$, A. Schäfer ${ }^{2}$

Hochschule für Angewandte Wissenschaft und Kunst, HAWK Hildesheim, Studiengang MSC ELP, Hildesheim

2 Hochschule Bremen, Fakultät 3 - Gesellschaftswissenschaften, Studiengang Angewandte Therapiewissenschaften Logopädie und Physiotherapie (ATW), Bremen

\section{Schlüsselwörter}

- Straight-Leg-Raise-Test (SLR)

- Änderungssensitivität

- kleinster klinisch relevanter Unterschied (MCIC)

\section{Key words}

- straight leg raise test (SLR)

- sensitivity to change

- minimum clinically important change (MCIC)

eingereicht 28.12 .2016

akzeptiert 1.3 .2017

\section{Bibliografie}

Dol https://doi.org/

10.1055/s-0035-1567228

physioscience 2017; 13:

162-169 @ Georg Thieme

Verlag KG Stuttgart . New York . ISSN 1860-3092

\section{Korrespondenzadresse}

Michael Bräuer, PT, MT, MSc,

\section{OMT-dvmt}

Hochschule für Angewandte Wissenschaft und Kunst,

HAWK Hildesheim,

Studiengang MSC ELP

Goschentor 1

31134 Hildesheim

mischa.braeuer@gmx.de

\section{Zusammenfassung}

\section{$\nabla$}

Hintergrund: Der Straight-Leg-Raise-Test (SLR) dient klinisch häufig zum Wiederbefund. Der kleinste klinisch relevante Unterschied (Minimal clinically important change, MCIC) und die Änderungssensitivität sind unbekannt.

Ziel: Diese longitudinale multizentrische Studie untersuchte den MCIC und die Änderungssensitivität für den Straight-Leg-Raise bei Patienten mit subakuten/akuten unteren Rückenschmerzen (LBP) und/oder unilateralen Beinschmerzen. Methode: Bei den eingeschlossenen 10 Probanden mit ausstrahlenden LBP wurden zu 2 Zeitpunkten ( 1 1; $\mathrm{t} 2)$ der SLR beidseitig mit/ohne Dorsalextension, der LBP sowie die unilateralen Beinschmerzen und die Aktivitätseinschränkung gemessen. Zu t2 wurden die subjektive Veränderung des Gesundheitszustands mittels der Global Rating of Change Scale (GROC) und Korrelationen zwischen den Veränderungen im SLR und der genannten Parameter ermittelt. Mithilfe der Area under the Curve (AUC) und der Korrelationen wurden die Änderungssensitivität und die Schwellenwerte für den SLR berechnet.

Ergebnisse: Die Veränderung des LBP zeigte gute bis sehr gute Korrelationen mit dem SLR mit/ohne Dorsalextension. Die AUC mit LBP als Anker erreichte im SLR einen fast akzeptablen Wert. Der dazugehörige Schwellenwert lag bei $17^{\circ}$.

Schlussfolgerung: Die zu kleine Stichprobengröße ließ keine präzisen Aussagen zu. Es gibt jedoch Hinweise, dass der SLR als änderungssensitiv für die Konstrukte unilaterale Beinschmerzen und LBP gelten könnte.

\section{Einleitung}

Beschwerden des unteren Rückens haben eine Lebenszeitprävalenz von 75 - 85\%. Damit handelt es sich um die am häufigsten auftretenden Beschwer-

\section{Abstract \\ $\nabla$}

Background: The straight leg raise test is clinically often used for reassessment. The minimum clinically important change (MCIC) and the sensitivity to change are unknown.

Objective: This longitudinal multicentre study investigated the MCIC and the sensitivity to change for the SLR in patients with subacute/acute low back pain (LBP) and/or unilateral leg pain.

Method: At 2 points in time (t1; t2) the bilateral SLR with/without dorsal extension, the LBP as well as the unilateral leg pain and the restriction of activity were assessed in the included 10 subjects with radiating LBP. At $\mathrm{t} 2$ the subjective change of physical health state was measured using the global rating of change scale (GROC). Correlations between SLR changes and the mentioned parameters were assessed. The SLR's sensitivity to change and threshold levels were calculated using the area under the curve (AUC).

Results: SLR change showed good to very good correlations with SLR with/without dorsal extension. AUC with LBP as anchor produced an almost acceptable performance. The corresponding threshold level was $17^{\circ}$.

Conclusions: The too small sample allowed no concise statement. There exists, however, an indication that the SLR might be considered to be sensitive to change for unilateral leg pain constructs.

den bei Patienten unter 45 Jahren [18]. Neben einer Vielzahl anderer Assessments kommt beim Untersuchungsprozess häufig der SLR zum Einsatz [11]. Er ist einer der meistverwendeten Tests zur Beurteilung lumbosakraler Nervenwurzelirritatio- 
nen [1], wie sie unter anderem durch einen Bandscheibenvorfall verursacht werden können [27].

Nach Nee und Butler [21] deutet ein positiver Test auf eine Steigerung der Mechanosensitivität hin, lässt aber keine Rückschlüsse auf den Ort der Läsion im Nervensystem zu. Die Validität des SLR wurde oft mittels des Goldstandard Magnetresonanztomografie (MRT) auf Vorliegen eines Bandscheibenvorfalls überprüft $[6,18,24]$. Dabei schwanken die angegebenen Werte zur Validität sehr stark. Für die Sensitivität liegen Werte zwischen 0,15 [19] und 0,95 [16] und für die Spezifität zwischen 0,14 [16] und 0,95 [18] vor. Es ist zu berücksichtigen, dass Studien zur Validität des SLR im physiotherapeutischen Setting fehlen und lediglich ärztliche Diagnosen (Bandscheibenvorfall) verwendet wurden.

Neben der Validität stellt die Fähigkeit von Messinstrumenten, eine Veränderung über die Zeit feststellen zu können (Änderungssensitivität), ein wichtiges Gütekriterium dar [20]. Da der SLR häufig zum Wiederbefund oder als Endpunkt der physiotherapeutischen Intervention benutzt wird [11], sollte nach Meinung der Autoren dessen Änderungssensitivität und MCIC bekannt sein.

Ziel der vorliegenden Studie war es zu untersuchen, ob Veränderungen des SLR mit Veränderungen anderer, krankheitsbezogener Merkmale einhergehen. Dies soll Aufschluss über die Diskriminationsfähigkeit des SLR geben. Weiterhin sollte festgestellt werden, wie groß eine Veränderung sein muss, um von den Patienten als wichtige Veränderung im individuellen Beschwerdebild wahrgenommen zu werden (Patient-reported outcome measures, PRO).

\section{Methode}

\section{Studiendesign und methodisches Vorgehen}

Zur Bestimmung der Änderungssensitivität wurde ein longitudinales Prä-post-Design mit 2 Messzeitpunkten (t1, t2) und Außenkriterium gewählt ( Abb.1). Um die Wahrscheinlichkeit zu erhöhen, die errechnete Probandenzahl von N=34 (ausgehend von einem 2-seitigen t-Test, einem Mittelwert der Differenz zwischen den Gruppen von $10 \pm 10$, beruhend auf der Annahme der Autoren, dass diese Differenz in einem klinischen Setting sicher zu messen sei und einem Alpha von 0,05 bzw. einem Beta von 0,8 ) zu erreichen, entschieden sich die Autoren für ein multizentrisches Vorgehen. Für die beiden Messzeitpunkte wurde basierend auf den klinischen Erfahrungen der Autoren über die mittlere Verweildauer von Patienten mit subakuten/akuten LBP in einer Praxis und zur Minimierung der Drop-out-Rate ein Abstand von 4-5 Wochen bestimmt. Dabei fanden folgende Messungen statt: SLR [5], visuelle Analogskala (VAS; [26]) und Roland Morris Disability Questionnaire (RMDQ) [8, 25]. In der Zwischenzeit wurden die Probanden physiotherapeutisch behandelt.

Zum Messzeitpunkt t2 wurde zusätzlich der Global-Rating-ofChange-Wert (GROC) erhoben. Dieser dient neben VAS und RMDQ als primäres Außenkriterium, d.h. als SLR-unabhängiges Messinstrument, um eine klinisch relevante Veränderung der subjektiv empfundenen Gesundheit festzustellen. Bei Probanden mit einem GROC größer 2 wurde von einer klinisch relevanten Verbesserung des empfundenen Gesundheitszustands ausgegangen, während ein GROC zwischen +2 und -2 keine Veränderung bedeutete. GROC-Werte kleiner als -2 deuten auf eine klinisch relevante Verschlechterung hin [13]. Diese Außenkriterien dienten dazu, die Probanden in Subgruppen mit und ohne klinisch relevante Veränderung einzuteilen.

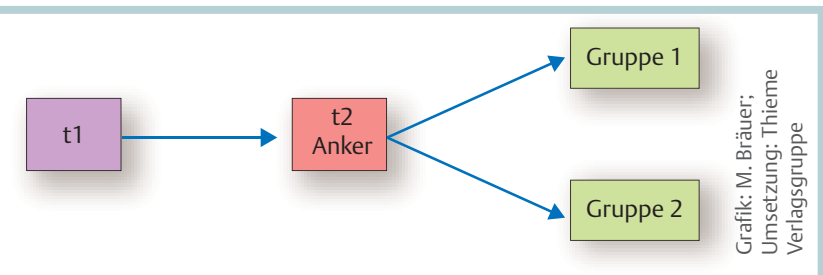

Abb. 1 Studiendesign.

\section{Teilnehmer}

Einschlusskriterien

mit subakuten/akuten LBP;

mit und ohne Ausstrahlung in ein Bein;

dorsaler Beinschmerz ohne LBP;

positiver SLR zum 1. Messzeitpunkt

Der SLR galt als positiv, wenn die in der Anamnese geschilderten für die Patienten typischen Symptome im Sinne von P1 reproduziert wurden. Sie mussten sich durch eine sensibilisierende distale Gelenksbewegung (Dorsalextension des Fußes) verändern lassen $[3,4]$.

\section{Ausschlusskriterien}

- Akute, starke und irritierbare Beschwerden: Dies bedeutet, eine leichte Aktivität in einer Bewegungsrichtung führt zu einem starken Schmerz, sodass die Aktivität abgebrochen werden muss und der Schmerz langsam abklingt [17]. Die entsprechende Einteilung in diese klinische Gruppe basiert anhand gewonnener Informationen aus der Anamnese und besonders aus dem 24-Stunden-Verhalten.

Neurologische Zeichen (Taubheit, nicht vorhandene Reflexe, Kraftminderung);

- Einnahme von Schmerzmedikamenten;

- Kontraindikation des SLR aus anderen Gründen, wie z. B. akut aufgetretene neurologische Symptome mit instabilem Verhalten, Läsionen der Cauda equina, Tethered Cord Syndrome

- Verdacht einer vorliegenden zentralen Sensibilisierung, da der SLR die Mechanosensitivität testet und es unter diesen Umständen gegebenenfalls Abweichungen gegenüber Patienten ohne zentrale Sensibilisierung geben könnte. Als Hinweise darauf wurden diffuse, ausgedehnte Schmerzen und empfindliche Zonen an mehreren Stellen gewertet. Lag ein unklares klinisches Bild vor, passte das Ausmaß der physischen Befunde nicht zum Behinderungsgrad und kam es zu einer Beeinflussung der Symptome durch affektive, kognitive und/oder psychosoziale Faktoren wurden eine zentrale Sensibilisierung vermutet.

\section{Teilnehmende Therapeuten}

Die Probanden wurden zwischen Januar 2015 bis Ende Juni 2015 im Rahmen einer Master-Arbeit an der HAWK-Hildesheim rekrutiert. Die teilnehmenden Therapeuten $(n=5)$ untersuchten ihre Patienten im laufenden Praxisalltag. Sie verfügten alle über eine mehrjährige Berufserfahrung ( $>4$ Jahre), 4 befanden sich in der Ausbildung zum OMT-dvmt und hatten bereits postgraduierte Kurse im Bereich manueller Therapie erfolgreich absolviert. Alle arbeiteten in ambulanten kleinen bis mittelgroßen Praxen.

\section{Durchführung des SLR}

Um einen reibungsfreien Ablauf zu gewährleisten und mögliche Fehler im Vorfeld zu erkennen, wurde der Testablauf vorab erprobt und die teilnehmenden Therapeuten in einer Schulung ent- 
sprechend instruiert. Die Schulung dauerte ca. 3 Stunden und fand mit allen Therapeuten gleichzeitig statt. Der Studienleiter demonstrierte den Ablauf. Anschließend übten alle Therapeuten die Durchführung des Messablaufs sowie die Handhabung des Messinstruments und der Studienunterlagen unter Supervision. Aufkommende Fragen wurden sofort in der Gruppe beantwortet und korrigiert.

Zur Messung der Hüftflexion beim SLR diente ein elektronisches Goniometer mit einem Neigungssensor für $360^{\circ}$-Messungen mit einer Genauigkeit von $\pm 0,1^{\circ}$. Es war auf einer mit Schaumstoff unterklebten Holzleiste angebracht, die an beiden Enden mittels Klettverschlussriemen am Unterschenkel der Probanden befestigt wurde ( Abb. 2). Die Patienten lagen auf dem Rücken, wobei der Kopf flach auf der Bank ruhte und die Hände locker auf dem Bauch lagen. Der Therapeut verhinderte die Kniebeugung (Endstellung Knieextension, hartes Endgefühl) durch einen flächigen Griff anterior auf dem distalen Oberschenkel. Die andere Hand befand sich unter dem distalen Ende des Unterschenkels der Patienten und führte das Bein ohne Hüftinnen- oder -außenrotation in die Hüftflexion. Das so gestreckte Bein wurde bis zum Einsetzen der Symptome (P1) der Patienten angehoben (SLR). Der Vorgang wurde mit adaptiertem Griff mit der Voreinstellung des Fußes in Dorsalextension (sensibilisierende Bewegung) wiederholt (DE-SLR).

Das Vorgehen erfolgte sowohl an der symptomatischen (Sy) als auch der asymptomatischen Seite (ASy). Die verbale Beschreibung auf der Sy-Seite lautete: „Dies ist ein Test, der die Beweglichkeit ihres Hüftgelenks mit gestrecktem Bein prüft. Ich hebe nun Ihr gestrecktes Bein an und Sie sagen stopp, wenn Ihre typischen Beschwerden im Rücken, Gesäß oder Bein anfangen. Sollten andere Symptome auftreten, die für Sie schmerzhaft sind, sagen Sie ebenfalls stopp und auf der ASy-Seite: „Dies ist ein Test, der die Beweglichkeit ihres Hüftgelenks mit gestrecktem Bein prüft. Ich hebe nun Ihr gestrecktes Bein an und Sie sagen stopp, wenn die Wahrnehmung von Dehnung anfängt, unangenehm zu werden."

Daraus resultierten folgende Messwerte:

- SLR ohne DE zum Zeitpunkt t1 (SLR_t1) bzw. t2 (SLR_t2);

- SLR mit DE zum Zeitpunkt t1 (DE_SLR_t1) bzw. t2 (DE_SLR_t2). Zum 1. Messzeitpunkt wurden auch die demografischen Werte der Patienten erhoben.

\section{Visuelle Analogskala (VAS)}

Bei der VAS trägt der Patient die empfundene Schmerzintensität auf einer $100 \mathrm{~mm}$ langen Linie ab. Dabei entspricht der linke Anker der Skala der Aussage „kein Schmerz “ und der rechte Anker stellt den „stärksten Schmerz dar, den sich der Patient vorstellen kann“ [10]. Zur Auswertung wird der Abstand vom linken Rand der Skala bis zur Markierung des Patienten gemessen. Als MCIC wurde der von Ostelo et al. [22] vorgeschlagene Wert von $15 \mathrm{~mm}$ verwendet. Über das Skalenniveau der VAS gibt es widersprüchliche Angaben: Kersten et al. [15] postulieren eine Ordinalskala, wohingegen Price et al. [23] auch die Verwendung parametrischer Tests vorschlagen.

\section{Roland Morris Disability Questionnaire (RMDQ)}

Da sich der RMDQ gut bei Patienten mit milden bis moderaten Beschwerden einsetzen lässt, wurde er für die hier zu betrachtende Population dem Oswestry Disability Index (ODI) vorgezogen [25]. Beim RMDQ kommt es bei starken Beeinträchtigungen zu Deckeneffekten, während der ODI noch Änderungen zeigen kann [25]. Da dies nur bei einer Population mit akuten, starken und irritierbaren Beschwerden zum Tragen kommt, hier aber

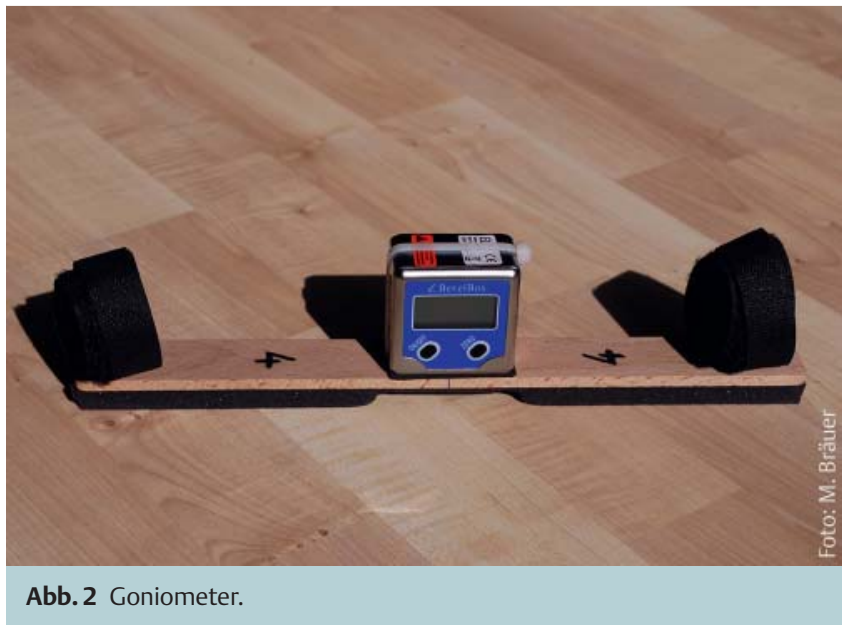

lediglich Probanden mit subakuten/akuten, nicht irritierbaren Beschwerden eingeschlossen wurden, überwogen die Vorteile des RMDQ.

Ein weiterer Grund für die Verwendung des RMDQ war das Vorliegen einer deutschen Version [8]. Roland und Fairbank [25] schlagen für den RMDQ im Rahmen von klinischen Studien zur Berechnung der Studienpopulationsgröße als MCIC eine Änderung von 2 - 3 Punkten vor. Die sehr gute Intratester-Reliabilität von 0,93 (Intraclass correlation coefficient, ICC) für Patienten mit LBP sprach ebenso für den RMDQ [12].

\section{Global Rating of Change (GROC)}

Global-Rating-of-Change-Skalen (GROC) kommen häufig in klinischen Studien zu muskuloskeletalen Fragestellungen zum Einsatz [13]. Die Skalen fragen nach der Einschätzung der Veränderung des empfundenen Gesundheitszustands von Patienten zwischen 2 Zeitpunkten [13]. Die Patienten beantworten die Frage mit einem Wert auf einer Likert-Skala. Als Empfehlung gilt eine 11Punkte Skala, die von -5 (sehr viel schlechter) bis +5 (komplett erholt im Sinne von keine Beschwerden mehr) reicht. 0 steht für keine Veränderung. Diese Skala diente als primäres Outcome ( Abb.3). Den Patienten wurde folgende Frage gestellt: „Im Hinblick auf Ihre Beschwerden im Bereich des unteren Rückens sowie (falls zutreffend) der Beinschmerzen und der damit verbundenen Beeinträchtigungen in Ihrem Alltag, wie würden Sie Ihren Zustand im Augenblick beschreiben, im Vergleich zu dem Zeitpunkt als Sie das 1 . Mal bei mir waren?"

Die GROC-Skala besitzt eine hohe Augenscheinvalidität. Ihr MCIC beträgt 2 [9, 13]. Deshalb wurden Probanden mit einem Wert größer +2 in die Gruppe mit einer signifikanten Verbesserung, Probanden mit einem Wert zwischen +2 und -2 in die Gruppe mit keiner Veränderung und die mit einem Wert schlechter als -2 in die Gruppe mit einer Verschlechterung eingeteilt.

Auch hier entschieden sich die Autoren für dieses Assessment aufgrund der sehr guten Intrarater-Reliabilität von ICC $=0,9$ und der hohen Augenscheinvalidität für Patienten mit LBP [13].

\section{Ethik}

In Übereinstimmung mit der Deklaration von Helsinki [28] hatten alle Probanden vor Studienbeginn eine Einverständniserklärung unterschrieben, nachdem sie über den genauen Ablauf aufgeklärt wurden. Alle Teilnehmer hatten zu jedem Zeitpunkt die Möglichkeit, ihr Mitwirken ohne Konsequenzen zu beenden. Alle Daten wurden anonymisiert, sodass keine Rückschlüsse auf 


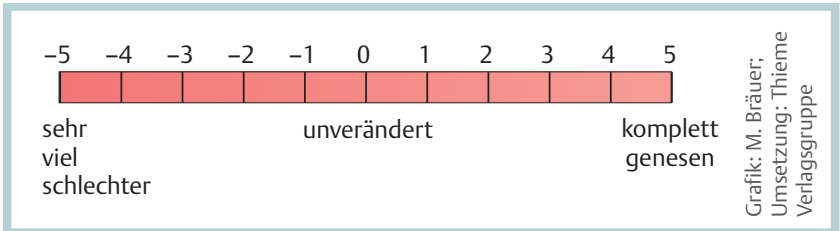

Abb. 3 Global Rating of Change Scale (GROC).

einzelne Personen möglich sind. Da die Studie lediglich Daten des Behandlungsprozesses erhob und nicht in diesen eingriff, wurde sichergestellt, dass alle Patienten die für sie notwendige Therapie erhielten. Zum Zeitpunkt der Durchführung der Studie gab es an der HAWK Hildesheim noch keine Ethikkommission, weshalb die Frage im Rahmen der „Research Conference“ besprochen wurde. Als Interventionsstudie stuften die eingeladenen Experten die Studie als ethisch unbedenklich ein.

\section{Analyse}

Nach Abschluss der Datenerhebung wurde die Studienpopulation anhand folgender, als gruppierende Variablen (Anker) verwendeter Parameter in 2 Subgruppen für die jeweiligen Konstrukte VAS, RMDQ oder GROC mit klinisch relevanter Veränderung ja (MCIC+) oder nein (MCIC-; Kontrollgruppe) eingeteilt: (1) VAS_LBP, (2) VAS_Bein, (3) GROC und (4) RMDQ ( $\bullet$ Abb. 1). Somit stellt die gruppierende Variable (Anker) in der ROC-Analyse die zu untersuchende „Diagnose“ dar.

Die Werte für die Einteilung waren wie folgt: VAS: $-15 \mathrm{~mm}$, RMDQ: -3 Punkte, GROC: - 2. Zur Ermittlung des Schwellenwerts des SLR für das Treffen dieser Diagnose wurde die Summe aus ([1-Sensitivität] + [1-Spezifität]) ermittelt. An der kleinsten Stelle wurde der Wert abgelesen. Die so generierten Subgruppen wurden sowohl deskriptiv dargestellt als auch auf signifikante Unterschiede in der Veränderung des SLR hin analysiert. Aufgrund der geringen Studienpopulation kam hier der Mann-Whitney-U-Test zum Einsatz [2]. Es wurden sowohl (1) die Intra-Beinveränderung (Veränderung des SLR innerhalb derselben Seite) als auch (2) die Inter-Beinveränderung (Veränderung zwischen den Seiten) im SLR evaluiert. Für (1) lautete die Frage, wie stark sich der Sy_SLR geändert hat und für (2), wie stark sich gegebenenfalls das vorher bestehende asymmetrische Bewegungsausmaß im SLR zwischen Sy_SLR und Asy_SLR aufhob.

Zur Berechnung der Änderungssensitivität dienten die Differenzwerte [7]. Darunter ist der Unterschied der gleichen Konstrukte (VAS, SLR, RMDQ) zwischen den beiden Zeitpunkten zu verstehen. Für die Bestimmung der Diskriminationsfähigkeit wurde die Area under the Curve (AUC, Fläche unter der ROC-Kurve) genutzt. Eine AUC von 0,7 gilt als akzeptabel [7].

Die Korrelationen der Differenzwerte der SLR-Varianten wurden jeweils mit den Differenzen der VAS und des RMDQ sowie mit dem GROC-Wert anhand des Spearman Korrelationskoeffizienten ermittelt. Für die Berechnungen wurde SPSS 19 eingesetzt.

\section{Ergebnisse}

Trotz multizentrischem Design musste wegen der begrenzten zeitlichen Ressourcen bei dieser Arbeit von der zuvor berechneten Samplesize abgewichen werden. Aufgrund der kleinen Stichprobe wurde im deskriptiven Teil der Median als Lagemaß verwendet.
Insgesamt konnten 10 Probanden eingeschlossen werden (6 Frauen, 4 Männer). Das Durchschnittsalter betrug im Median 32 Jahre. Im Mittel hatten die Probanden seit 4 Wochen Beschwerden im unteren Rücken und/oder Bein. Zum Zeitpunkt t1 erreichte die Schmerzintensität für den unteren Rücken $57 \mathrm{~mm}$ auf der VAS und die Schmerzen im Gesäß bzw. Bein - falls vorhanden - 32,5 mm. Zum Zeitpunkt t2 lagen die Rückenbeschwerden mit einer mittleren Intensität auf der VAS bei $4,5 \mathrm{~mm}$. Der Beinschmerz reduzierte sich im Median auf $0 \mathrm{~mm}$.

Der Unterschied zum Zeitpunkt t1 im SLR zwischen der Symptom reproduzierenden Seite (positiver SLR) und der Symptom nicht reproduzierenden Seite (negativer SLR) betrug $18^{\circ}(\mathrm{p}=0,02)$ und $1^{\circ}$ zum Zeitpunkt t2 ( $p=0,14$; $\vee$ Tab. 1 ). Für den DE-SLR zum Zeitpunkt t1 lag der Unterschied bei $8,5^{\circ}(p=0,03)$ und $0^{\circ}(p=0,15)$ zum Zeitpunkt t2. Auf der Seite des positiven SLR betrug der Unterschied beim SLR $13,5^{\circ}(p=0,07)$ zwischen den beiden Messzeitpunkten und $9^{\circ}(\mathrm{p}=0,08)$ mit dem DE-SLR. Auf der Seite des negativen SLR erzielte der Unterschied beim SLR $-2,5^{\circ}(p=0,60)$ zwischen den beiden Messzeitpunkten und $-1,50(p=0,74)$ mit dem DE-SLR. Die Patienten gaben im Schnitt einen GROC-Wert von 3,5 an. Zwischen $\mathrm{t} 1$ und $\mathrm{t} 2$ vergingen im Median 35,5 Tage, und die Patienten erhielten 6 Behandlungen.

Im RMDQ zu t1 wurde ein Score von 4,5 im Vergleich zu 2 zum Zeitpunkt t2 erhoben. Der Unterschied für den RMDQ zwischen den beiden Messpunkten betrug 2,5 Punkte $(p=0,09)$.

Im Median verringerte sich die Schmerzintensität für den Rückenschmerz von t1 zu t2 um $25,5 \mathrm{~mm}(\mathrm{p}=0,03)$ und für den Beinschmerz um 26,5 $5^{\circ}(p=0,02)$.

Zur Bestimmung der Änderungssensitivität wurde geprüft, ob die Veränderung der Variablen VAS, RMDQ und dem GROC mit der Veränderung im SLR korrelierten. Die Berechnungen erfolgten aufgrund fehlender Normalverteilung mittels Spearmans Rangkorrelation ( $\bullet$ Tab.2). Gute Korrelationen fanden sich zwischen der Änderung des LBP und der Änderung im SLR $(r=-0,74)$ mit $\mathrm{p}=0,02($ Abb.4) und dem DE-SLR $(\mathrm{r}=-0,81)$ mit $\mathrm{p}<0,01$ ( Abb.5).

Die Korrelation des LBP und der Änderung des Unterschieds zwischen den Beinen ergab moderate Werte $(r=0,59)$ mit einem $\mathrm{p}=0,09$. Der Zusammenhang zwischen dem GROC und der Änderung des Unterschieds zwischen beiden Beinen im DE-SLR erzielte ebenfalls eine moderate Korrelation $(r=0,57)$ mit $p=0,08$.

Nach der Einteilung der Probanden in die bereits beschriebenen Subgruppen wurden Unterschiede im Bewegungsausmaß im SLR zwischen den Subgruppen analysiert $(\checkmark$ Tab.3). Der Gruppenvergleich zwischen einer klinisch relevanten und klinisch nicht relevanten Veränderung (Kontrollgruppe) bezogen auf den Rückenschmerz legte die Vermutung nahe, dass sich dieser im SLR abbilden ließe, da dieser mit p =0,07 Hinweise lieferte, in größeren Studien signifikant werden zu können. Für den Beinschmerz konnten ähnliche Rückschlüsse gezogen werden $(p=0,09)$. Die Ergebnisse der ROC-Analyse sind in $\bullet$ Tab. 4 dargestellt.

Bei der Analyse der AUC zur Beurteilung der Diskriminationsfähigkeit erwiesen sich SLR und DE-SLR mit einer AUC von 0,68 bis 0,86 für die Konstrukte VAS_Rücken, VAS_Bein und RMDQ als potenziell geeignetes Assessment zur Beurteilung der klinisch relevanten Veränderung von Beschwerden. Unter Berücksichtigung der ermittelten Korrelationen käme alleine der Schwellenwert für den SLR mit $17^{\circ}$ in Betracht. Er erzielte eine AUC von 0,679 $(\mathrm{p}=0,46 ; \sim$ Tab. 4$)$. 
Tab. 1 Übersicht der Studienergebnisse.

\begin{tabular}{|c|c|c|c|c|c|}
\hline Median (95\%-KI) & $N=10$ & t1 & t2 & \multicolumn{2}{|l|}{ p-Wert } \\
\hline Alter & 32 Jahre $(24,3 ; 48,7)$ & & & & \\
\hline Geschlecht & 6 Frauen/4 Männer & & & & \\
\hline Symptomdauer & 4 Wochen & & & & \\
\hline $\operatorname{LBP}(n)$ & & 9 & 5 & & \\
\hline Beinschmerz ( $n$ ) & & 9 & 4 & & \\
\hline RMDQ (Punkte) & & $4,5(2,6 ; 13,4)$ & $2(0 ; 10,7)$ & $\begin{array}{l}0,086 \text { (2-seitig) } \\
0,043^{1} \text { (1-seitig) Wi }\end{array}$ & -Rangsummentest \\
\hline Diff_RMDQ (Punkte) & & $2,5(-0,7 ; 5,7)$ & & & \\
\hline VAS_Rücken (mm) & & $57(10,3 ; 61)$ & $4,5(0 ; 32,1)$ & $0,026^{1}$ verbundene & \\
\hline Diff_VAS_Rücken (mm) & & $-25,5(-55,4 ; 2,7)$ & & & \\
\hline VAS_Bein $(\mathrm{mm})$ & & $32,5(7,6 ; 75,7)$ & $0(0 ; 10,5)$ & $0,022^{1}$ verbundene & \\
\hline Diff_VAS_Bein (mm] & & $-26,5(-75 ;-1,6)$ & & & \\
\hline GROC (Punkte) & & & $3,5(2 ; 5)$ & & \\
\hline Zeitdifferenz t1-t2 (Tage) & & 35,5 & & & \\
\hline Anzahl Behandlungen (n) & & $6(6 ; 8)$ & & & \\
\hline SLR $\times$ Median $(95 \%-K I)$ & & & & $\begin{array}{l}\text { Diff. Sy-ASy t1 } \\
\text { p-Wert (1) \& (2) }\end{array}$ & $\begin{array}{l}\text { Diff. Sy-ASy t2 } \\
\text { p-Wert t2 (1) \& (2) }\end{array}$ \\
\hline symptomauslösend $\left(x^{\circ}\right)(1)$ & & $42(22,8 ; 54,7)$ & $54(50 ; 67,7)$ & $18(5 ; 36,4)$ & $1(-2 ; 12,7)$ \\
\hline nicht symptomauslösend $\left[\mathrm{x}^{\circ}\right](2)$ & & $63(45,2 ; 86,1)$ & $60(53 ; 78,8)$ & $0,018^{1}$ & 0,144 \\
\hline Differenz t2-t1 $\left[\mathrm{x}^{\circ}\right](1)$ & & $13,5(-2,4 ; 30,5) p$ & 0,07 & & \\
\hline Differenz t2-t1 [ $\left.\mathrm{x}^{\circ}\right](2)$ & & $-2,5(-12,7 ; 6,7) p$ & 0,6 & & \\
\hline DE-SLR × Median (95\%-KI) & & & & p-Wert t1 (3) \& (4) & p-Wert t2 (3) \& (4) \\
\hline symptomauslösend $\left[\mathrm{x}^{\circ}\right](3)$ & & $33(13,1 ; 45,4)$ & $47(36 ; 53,7)$ & $8,5(3 ; 52,2)$ & $0(-2 ; 16,7)$ \\
\hline nicht symptomauslösend $\left[\mathrm{x}^{\circ}\right](4)$ & & $55(37,3 ; 74,8)$ & $51,5(43,3 ; 66,7)$ & $0,027^{1}$ & 0,152 \\
\hline Differenz t2-t1 $\left[\mathrm{x}^{\circ}\right](3)$ & & $9(-1,7 ; 33,5) p=0$ & & & \\
\hline Differenz t2-t1 $\left[\mathrm{x}^{\circ}\right](4)$ & & $-1,5(-9,4 ; 8,7) p=$ & & & \\
\hline
\end{tabular}

ASy = asymptomatisch; DE = Dorsalextension; Diff_RMDQ = Differenz im RMDQ zwischen $\mathrm{t} 1$ und t2; Diff_VAS_Rücken/Bein = Differenz der VAS zwischen t1 und t2 bezogen auf den Rücken- und Beinschmerz; GROC= Global Rating of Change Scale; LBP = Low Back Pain; SLR =Straight Leg Raise Test; RMDQ = Roland Morris Disability Questionnaire; Sy= symptomatisch; $\mathrm{t} 1$ = Messzeitpunkt $1 ; \mathrm{t} 2$ = Messzeitpunkt 2 .

$1 \mathrm{p}$-Wert $\leq 0,05$

Tab. 2 Korrelation der Veränderung im Straight Leg Raise Test und den Veränderungen in der visuellen Analogskala (Ordinalskala), Roland Morris Disability Questionnaire und Global Rating of Change Scale über die Zeit ( $n=10$; alle Angaben beziehen sich auf die jeweilige Veränderung zwischen $t 1$ und $t 2)$.

\begin{tabular}{|c|c|c|c|c|}
\hline & \multicolumn{4}{|c|}{ Unterschied zwischen $\mathrm{t} 1$ und $\mathrm{t} 2$} \\
\hline & \multicolumn{4}{|l|}{ Diff_VAS } \\
\hline & Rücken a) & Bein b) & Diff_RMDQ & GROC \\
\hline Diff_SLR_Sy & $-0,74(p=0,02)^{1}$ & $-0,502(p=0,16)^{2}$ & $-0,515(p=0,13)^{2}$ & $-0,48(p=0,16)$ \\
\hline Diff_SLR_DE_Sy & $-0,81(p<0,01)^{1}$ & $-0,46(p=0,20)$ & $-0,511(p=0,13)^{2}$ & $-0,47(p=0,17)$ \\
\hline Diff_SLR_Sy_ASy & $0,328(p=0,38)$ & $0,477(p=0,19)$ & $0,081(p=0,82)$ & $0,47(p=0,16)$ \\
\hline Diff_SLR_DE_Sy_ASy & $0,593(p=0,09)^{2}$ & $0,542(p=0,13)^{2}$ & $0,11(p=0,76)$ & $0,574(p=0,08)^{2}$ \\
\hline
\end{tabular}

a) Patienten ohne Rückenschmerz zu t1 wurden ausgeschlossen ( $n=9$ ); b) Patienten ohne Beinschmerz zu t1 wurden ausgeschlossen ( $n=9$ ); ASy= asymptomatisch; DE= Dorsalextension; Diff_RMDQ = Differenz im RMDQ zwischen t1 und t2; Diff_VAS=Differenz der VAS zwischen t1 und t2; GROC=Global Rating of Change Scale; RMDQ = Roland Morris Disability Questionnaire; SLR = Straight Leg Raise Test; Sy= symptomatisch; t1 = Messzeitpunkt 1; t2 = Messzeitpunkt 2; VAS = visuelle Analogskala ${ }^{1} \mathrm{p}<0,05$;

2 Werte, welche eine mindestens mittlere Korrelation zeigen $(\geq 0,5)$ und bei größerer Stichprobe am ehesten signifikant werden könnten

\section{Diskussion}

Aufgrund der geringen Probandenzahl verzichteten die Autoren bei dieser explorativen Pilotstudie zur Berechnung der Korrelationen auf den ICC. Eine klare Aussage über konkrete Zusammenhänge und Signifikanzen ist an dieser Stelle leider nicht möglich. Daher sind alle Ergebnisse als Hinweise zu verstehen, um größere Studien besser planen zu können.

Eine gute Korrelation erreichte der Unterschied zwischen den Messzeitpunkten im DE- und SLR in Bezug auf die VAS-Veränderung für den Rückenschmerz. Die Korrelation zwischen dem Un- terschied im DE-SLR und dem Rückenschmerz war mit $r=0,81$ $(\mathrm{p}<0,01)$ stark. In Bezug auf den GROC ergaben sich durchweg mittlere bis gute Korrelationen. Damit wäre zu vermuten, dass die Aufhebung der Veränderung im Unterschied zwischen den Beinen im Median von 8,5 $(\mathrm{t} 1)$ auf $0^{\circ}(\mathrm{t} 2)$, gemessen mittels dem DE-SLR ( Tab.1) am ehesten die subjektiv eingeschätzte Veränderung der Beschwerden (GROC) abbilden könnte.

Ähnliche Aussagen ließen sich über den Zusammenhang gegenüber dem LBP treffen. Ob jedoch eher die Änderung auf der SLRpositiven Seite oder die Verringerung der Differenz zwischen den Beinen eine bessere klinische Aussage über Schmerzintensität 


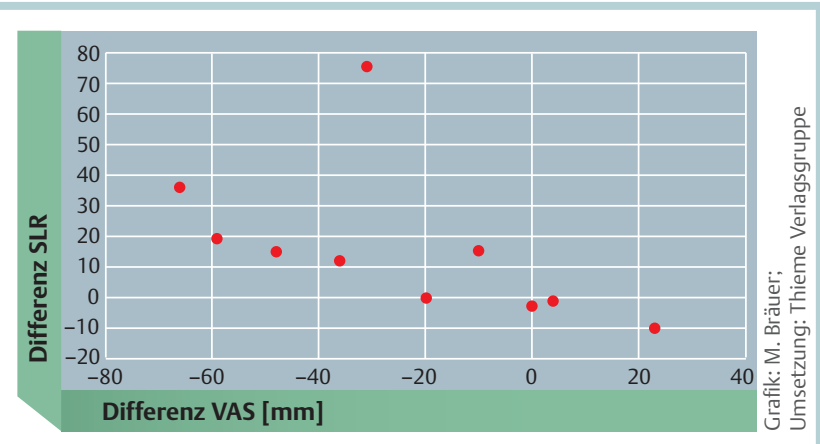

Abb. 4 Zusammenhang der Änderung im Straight Leg Raise Test (SLR) und der visuellen Analogskala (VAS) für Low Back Pain (LBP).

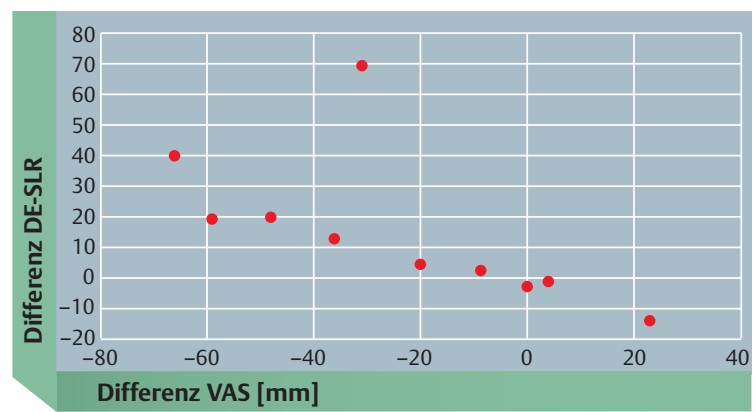

Abb. 5 Zusammenhang der Änderung Straight Leg Raise Test in Dorsalextension (DE-SLR) und der visuellen Analogskala (VAS) für Low Back Pain (LBP).

bzw. subjektive Beurteilung der Beschwerden darstellt, ist unklar. Da aber die Patienten im Mittelpunkt der Therapie stehen, ist die Beurteilung der Veränderung des Unterschieds zwischen den Beinen mit dem DE-SLR klinisch tendenziell eher zu empfehlen.

Der Beinschmerz zeigte moderate Korrelation mit dem SLR ( $p>0,05)$, sowohl im Unterschied des SLR eines Beines als auch im Unterschied über die Zeit im Vergleich der Beine. Eine mögliche Erklärung könnte sein, dass die Beinschmerzen meist im Zusammenhang mit den Rückenschmerzen auftraten. Lediglich ein Proband wies Beinschmerzen ohne Rückenschmerzen auf, die durch den SLR ausgelöst werden konnten. Ein weiterer Grund könnte sein, dass die Ausschlusskriterien die Aufnahme von Probanden mit neurologischen Zeichen verhinderten. Demzufolge ist zu vermuten, dass es sich bei dem Beinschmerz eher um Referred pain der LWS handelte.

Für die Einteilung anhand der klinisch relevanten Veränderung im GROC-RMDQ fand sich kein möglicher Unterschied bezogen auf den SLR beider Gruppen, da 9 der 10 Probanden angaben, sich klinisch relevant gebessert zu haben und lediglich ein Proband seine Beschwerden als „schlechter“ einstufte. Probanden ohne klinisch relevanten Unterschied gab es nicht, sodass sich keine Schwellenwerte für den SLR ziehen ließen.

Zur Bestimmung des MCIC bezüglich der hier erfassten Konstrukte wurden nur die Messungen betrachtet, die einen Trend zu einem signifikanten Unterschied zeigen könnten. Die 1. Betrachtung des Schwellenwerts bezog sich auf das Konstrukt

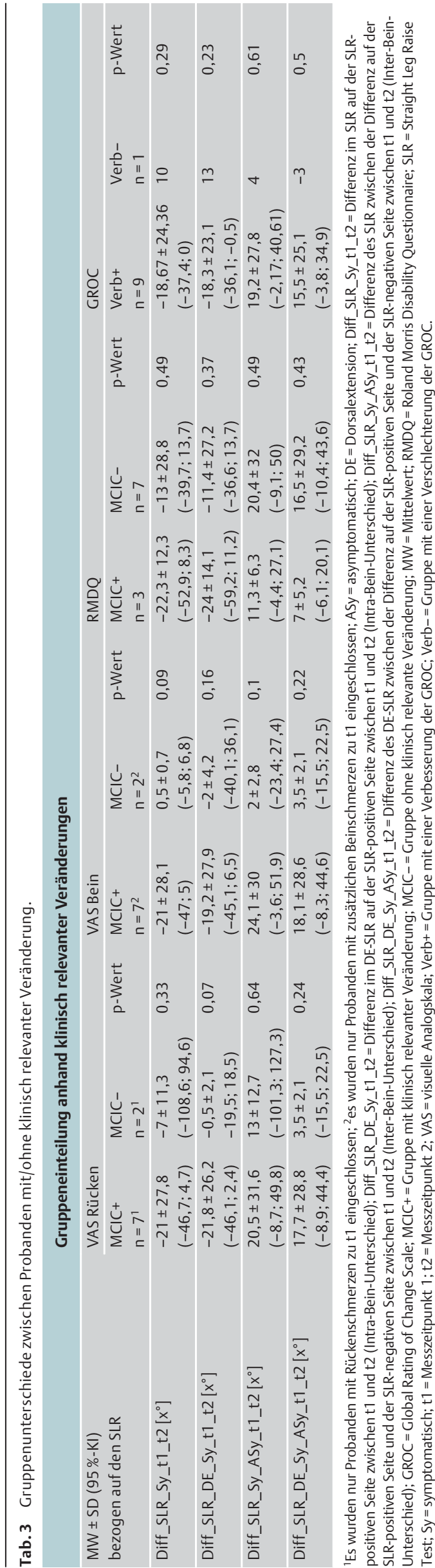


Tab. 4 Schwellenwert Straight Leg Raise Test für den kleinsten klinisch relevanten Unterschied im Konstrukt visuelle Analogskala, Roland Morris Disability Questionnaire und Global Rating of Change Scale.

\begin{tabular}{|c|c|c|c|c|c|c|c|c|c|c|c|c|}
\hline \multirow{3}{*}{$\begin{array}{l}\text { Unterschied } \mathbf{t} \text { 1-t2 } \\
\text { SLR }\end{array}$} & \multicolumn{12}{|c|}{ Variable } \\
\hline & \multicolumn{3}{|c|}{ VAS-Rücken ( $n=9)$} & \multicolumn{3}{|c|}{ VAS-BEIN $(n=9)$} & \multicolumn{3}{|c|}{ RMDQ $(n=10)$} & \multicolumn{3}{|c|}{ GROC $(n=10)$} \\
\hline & AUC & $\operatorname{SW}\left[\mathrm{x}^{\circ}\right]$ & p-Wert & AUC & $\operatorname{SW}\left[\mathrm{x}^{\circ}\right]$ & p-Wert & AUC & $\mathrm{SW}\left[\mathrm{x}^{\circ}\right]$ & p-Wert & AUC & $\operatorname{SW}\left[\mathrm{x}^{\circ}\right]$ & p-Wert \\
\hline Diff_SLR_Sy & 0,679 & 17 & 0,464 & 0,714 & 7,5 & 0,380 & 0,762 & 6 & 0,210 & 1,000 & 6,5 & 0,117 \\
\hline Diff_SLR_DE_Sy & 0,857 & 3,5 & 0,143 & 0,643 & 12 & 0,558 & 0,762 & 9 & 0,210 & 1,000 & 7,5 & 0,117 \\
\hline Diff_SLR_Sy_ASy & 0,571 & 18,5 & 0,770 & 0,036 & $1 \& 92$ & 0,057 & 0,476 & 18,5 & 0,909 & 0,222 & 2 & 0,384 \\
\hline Diff_SLR_DE_Sy_ASy & 0,286 & 56,5 & 0,380 & 0,156 & 0,5 & 0,242 & 0,524 & 16,5 & 0,909 & 0,000 & 50,5 & 0,117 \\
\hline
\end{tabular}

ASy = asymptomatisch; AUC = Area under the Curve; DE = Dorsalextension; Diff_SLR_PF_Sy= Unterschied im SLR auf der SLR-positiven Seite (Intra-Bein-Unterschied); Diff_SLR_DE_Sy= Unterschied im DE-SLR auf der SLR-positiven Seite (Inter-Bein-Unterschied); Diff_SLR_Sy_ASy= Unterschied des SLR zwischen der SLR-positiven und SLR-negativen Seite (Inter-Bein-Unterschied); Diff_SLR_DE_Sy_ASy= Unterschied des DE-SLR zwischen der SLR-positiven und SLR-negativen Seite (Inter-Bein-Unterschied); GROC= Global Rating of Change Scale; RMDQ = Roland Morris Disability Questionnaire; SLR = Straight Leg Raise Test; SW = Schwellenwert; Sy= symptomatisch; $\mathrm{t} 1=$ Messzeitpunkt 1; t2 = Messzeitpunkt 2; VAS = visuelle Analogskala.

Rückenschmerz. Da der Unterschied im DE-SLR von t1 zu t2 zwischen beiden Gruppen (MCIC+ und MCIC-) den geringsten pWert annahm $(p=0,07)$, wurde der dazugehörige Schwellenwert ermittelt. Mit einer akzeptablen AUC $(A U C=0,86)$ könnte sich hier eine gute Diskriminationsfähigkeit zeigen. Der Schwellenwert lag knapp über dem Messfehler der Messanordnung $\left(3^{\circ}\right)$. Der SLR erreichte dabei eine fast akzeptable Diskriminationsfähigkeit $(A U C=0,68)$. Der Schwellenwert von $17^{\circ}$ könnte für die klinische Praxis eine erste Orientierung geben.

\section{Limitierungen}

Eine große Limitierung der hier präsentierten Daten ist die kleine Stichprobe $(\mathrm{N}=10)$. Die Gründe hierfür sind vielfältig. Zum einen waren die Einschlusskriterien sehr eng gefasst und zum anderen die Anzahl an potenziellen Patienten in den jeweiligen Einrichtungen sehr gering.

Der Studienablauf fand neben dem Praxisalltag statt. Ein Therapeut arbeitete im 15-Minuten Rhythmus, was die Erhebung des SLR innerhalb einer Behandlungssitzung wesentlich erschwerte. Durch das longitudinale Studiendesign ist es notwendig, die Probanden nach 4 - 5 Wochen eventuell telefonisch zu kontaktieren. Zusätzlich erfordert dies auch eine hohe Bereitschaft von Seiten der Patienten, sich für die Datenerhebung erneut in die Praxis zu begeben, wobei für sie kein Nutzen entsteht. Einige Daten lassen vermuten, dass die erhobenen Werte biasbehaftet sind.

Der Rückenschmerz wurde separat zum Beinschmerz erhoben. Das bedeutet, Patienten mit Rücken- und Beinschmerz wurden 2 Werte zugeordnet. Welches der beiden Symptome der SLR auslöste und als P1 diente, floss nicht in die Datenerhebung ein. Nachfolgende Studien sollten durch den SLR ausgelöste und mit sensibilisierender Bewegung beeinflusste Symptome als das zu messende Konstrukt betrachten.

Die Sinnhaftigkeit der Trennung der Beschwerdebereiche ist auch zu diskutieren. Es müssten genauere Definitionen erstellt werden, ab welcher Stärke ein Beinschmerz als solcher zu bezeichnen ist (z. B. Schmerzen im Bereich Gesäß, distal des Gesäßes oder bis mindestens oberes Drittel dorsaler Oberschenkel). Ebenfalls ist fraglich, ob bei vermutetem Referred Pain beide Bereiche getrennt betrachtet werden müssen.

Ein weiterer limitierender Faktor ist die Antwortverschiebung (Response shift). Das bedeutet, Patienten bewerten ihre Situation zu verschiedenen Zeitpunkten unterschiedlich, was eine Repriorisierung (die Wichtigkeit von Symptomen erhält einen anderen Stel- lenwert) oder Skalenumkalibrierung (durch z. B. neue Schmerzerfahrungen können der ursprüngliche Schmerz anders bewertet und neue Maßstäbe zum Zeitpunkt t2 angesetzt werden) sein kann. Daher sollten in Folgestudien die Probanden die Messergebnisse vom ersten Messzeitpunkt einsehen.

Da der SLR die Mechanosensitivität des Nervensystems erfassen soll und dadurch eine Beurteilung zulässt, müssten psychosoziale Konstrukte miterfasst werden, weil sie die Sensibilisierung des gesamten Nervensystems und somit möglicherweise auch den SLR beeinflussen.

Eine weitere Limitierung der Studie, die die Interpretation der Ergebnisse erschwert, ist das Fehlen der Reliabilität für die hier verwendete Durchführung.

\section{Schlussfolgerungen \\ $\nabla$}

Die Eignung des SLR als Assessment zur Beurteilung der Veränderung von Beschwerden bei Patienten mit subakutem/akutem Rückenschmerz und/oder Beinschmerz kann anhand der vorliegenden Daten nicht beurteilt werden. Es gibt jedoch Hinweise, dass der SLR eine gute Änderungssensitivität für die subjektive Einschätzung der Beschwerden der Probanden sowie für das Konstrukt Rückenschmerz besitzen könnte. Eine erste vorsichtige Orientierung könnte der Schwellenwert von $17^{\circ}$ für das Konstrukt Rückenschmerz bieten, um eine Diskrimination bezogen auf eine klinische relevante Veränderung im Schmerz der Probanden zu dokumentieren.

Diese Pilotstudie liefert jedoch wichtige Anhaltspunkte zur Patientenrekrutierung, der Praktikabilität der Messinstrumente und den erwarteten Effekten, die für die Planung von Folgestudien wichtig sind.

Interessenkonflikt: Die Autoren geben an, dass kein Interessenkonflikt besteht.

\section{Literatur}

1 Airaksinen O, Brox JI, Cedraschi C et al. European guidelines for the management of chronic nonspecific low back pain. Eur Spine J 2006; 15: $36-38$

2 Bortz J, Lienert G. Kurzgefasste Statistik für die klinische Forschung. Leitfaden für die Verteilungsfreie Analyse kleiner Stichproben. Heidelberg: Springer; 2003

3 Boyd BS, Wanek L, Gray AT et al. Mechanosensitivity of the Lower Extremity Nervous System During Straight-Leg Raise Neurodynamic Tes- 
ting in Healthy Individuals. J Orthop Sports Phys Ther 2009; 39: 780 790

4 Boyd BS, Villa PS. Normal inter-limb differences during the straight leg raise neurodynamic test: a cross sectional study. BMC Musculoskelet Disord 2012; 13: 245

5 Butler DS. Mobilisation des Nervensystems. Berlin: Springer; 1998

6 Capra F, Vanti C, Donati $R$ et al. Validity of the Straight-Leg Raise Test for Patients with Sciatic Pain with or without Lumbar Pain Using Magnetic Resonance Imaging Results as a Reference Standard. J Manipulative Physiol Ther 2011; 34: 231-238

7 De Vet HCW. Reliability. In: De Vet HCW, Terwee CB, Mokkink LB et al. (eds) Measurement in Medicine. Cambridge: University Press; 2011

8 Exner V, Keel P. Measuring disability of patients with low-back pain validation of a German version of the Roland \& Morris disability questionnaire. Schmerz 2000; 14: $392-400$

9 Fischer D, Stewart AL, Bloch DA et al. Capturing the patient's view of change as a clinical outcome measure. JAMA 1999; 282: 1157-1162

10 Hilfiker R, Oesch P. Schmerzintensität: Numeric Rating Scale (NRS)/Visual Analogue Scale (VAS). In: Oesch P, Hilfiker R, Keller S et al. (Hrsg). Assessments in der Rehabilitation. Bern: Huber; 2011

11 Van den Hoogen HJ, Koes BW, Devillé $W$ et al. The inter-observer reproducibility of Lasègue's sign in patients with low back pain in general practice. Br J Gen Pract 1996; 46: 727 -730

12 Jacob T, Baras M, Zeev A et al. Low back pain: Reliability of a Set of Pain Measurement Tools. Arch Phys Med Rehabil 2001; 82: 735 - 742

13 Kamper SJ. Maher CG. Mackay G. Global Rating of Change Scales: A Review of Strengths and Weaknesses and Considerations for Design. J Man Manip Ther 2009; 17: 163-170

14 Kelly $A$. The minimum clinically significant difference in visual analogue scale pain score does not differ with severity of pain; 2001; 18 205-207

15 Kersten P. Küçükdeveci AA. Tennant A. The use of the visual analogue scale (VAS) in rehabilitation outcomes. J Rehabil Med 2012; 44: 609610

16 Kosteljanetz M. Bang F. Schmidt-Olsen S. The clinical significance of Straight-Leg-Raising (Lasègue's Sign) in the diagnosis of prolapsed Lumbar Disc - Interobserver Variation and Correleation with Surgical Finding. Spine (Phila Pa 1976) 1988; 13: 393 - 395

17 Maitland GD. Hengeveld E. Banks K et al. Maitland - Manipulation der Wirbelsäule. Heidelberg: Springer; 2008
18 Majlesi J. Togay H. Unalan $\mathrm{H}$ et al. The sensitivity and specificity of the slump and the straight leg raising tests in patients with lumbar disc herniation. J Clin Rheumatol 2008; 14: 87-91

19 Martin HD. Kivlan BR. Palmer IJ et al. Diagnostic accuracy of clinical tests for sciatic nerve entrapment in the gluteal region. Knee Surg Sports Traumatol Arthrosc 2014; 22: 882-888

20 Mokkink LB. Terwee CB. Patrick DL et al. The COSMIN study reached international consensus on taxonomy, terminology, and definitions of measurement properties for health-related patient-reported outcomes. J Clin Epidemiol 2010; 63: 737-745

21 Nee RJ. Butler DS. Management of peripheral neuropathic pain: Integrating neurobiology, neurodynamics, and clinical evidence. Physical Therapy in Sport 2006; 7: 36-49

22 Ostelo RW. Deyo RA. Stratford $P$ et al. Interpreting change scores for pain and functional status in low back pain: towards international consensus regarding minimal important change. Spine (Phila Pa 1976) 2008; 33: $90-94$

23 Price DD. Staud R. Robinson ME. How should we use the visual analogue scale (VAS) in rehabilitation outcomes? II: Visual Analogue Scales as ratio scales: an alternative to the view of Kersten et al. J Rehabil Med 2012; 44: 800-804

24 Rabin A. Gerszten PC. Karausky P et al. The sensitivity of the seated straight-leg raise test compared with the supine straight-leg raise test in patients presenting with magnetic resonance imaging evidence of lumbar nerve root compression. Arch Phys Med Rehabil 2007; 88: $840-843$

25 Roland M. Fairbank J. The Roland-Morris Disability Questionnaire and the Oswestry Disability Questionnaire. Spine (Phila Pa 1976) 2000; 25 $3115-3124$

26 Schomacher J. Gütekriterien der visuellen Analogskala zur Schmerzbewertung. physioscience 2008; 4: 125-133

27 Smith SA. Massie JB. Chesnut $R$ et al. Straight leg raising: Anatomical Effects on the Spinal Nerve Root without and with Fusion. Spine (Phila Pa 1976) 1993; 18: 992 - 999

28 World Medical Association (WMA). WMA Deklaration von Helsinki Ethische Grundsätze für die medizinische Forschung am Menschen; 2013, www.bundesaerztekammer.de/fileadmin/user_upload/Deklaration_von_Helsinki_2013_DE.pdf (22.12.2016) 